\title{
Normed algebras with involution
}

Vladimír Müller, ${ }^{1}$ Prague

ABSTRACT: We show that most of the theory of Hermitian Banach algebras can be proved for normed $*$-algebras without the assumption of completeness.

The condition $r(x) \leq p(x)$ for all $x$ (where $p(x)=r\left(x^{*} x\right)^{1 / 2}$ is the Pták function), which is essential in the theory of Hermitian Banach algebras, is replaced for normed *-algebras by the condition $r(x+y) \leq p(x)+p(y)$ for all $x, y$. In case of Banach $*$-algebras these conditions are equivalent.

\section{Introduction:}

The investigation of Banach algebras with involution and their representations in the algebra of bounded operators on a Hilbert space was started in 1943 by I. M. Gelfand and M. A. Naimark [3] and it was continued by many authors (see e.g. [4], [6], [8], [10], [11], [12]). The theory culminated by the following, already classical, results: characterization of $C^{*}$-algebras as those satisfying $\left\|x^{*} x\right\|=\|x\|^{2}$, the Vidav-Palmer theorem and the characterization of the Hermitian Banach algebras. All these results are closely connected to each other. A survey of the theory can be found in [9]. An important tool in the above mentioned results is the Ford square root lemma [2].

The aim of this paper is to show that, rather surprisingly, a great part of the theory can be proved without the assumption of completeness (which is of course necessary for existence of square roots, inverse elements etc.).

All algebras under consideration are complex and possess identity elements denoted by 1 . The set of real and complex numbers will be denoted by $\mathbf{R}$ and $\mathbf{C}$, respectively.

Let $A$ be a normed algebra with involution. Denote by $\widetilde{A}$ the completion of $A$ (we do not suppose the continuity of the involution so that in general it is not possible to extend the involution to $\widetilde{A}$ ). The spectral radius of an element $a \in A$ will be denoted by

$$
r(a)=\lim _{n \rightarrow \infty}\left\|a^{n}\right\|^{1 / n}=\max \left\{|\lambda|, \lambda \in \sigma_{\tilde{A}}(a)\right\}
$$

where

$$
\sigma_{\tilde{A}}(a)=\{\lambda \in \mathbf{C}, \lambda-a \text { is not invertible in } \widetilde{A}\} .
$$

The spectrum in the original algebra $\sigma_{A}(a)=\{\lambda \in \mathbf{C}, \lambda-a$ is not invertible in $A\}$ is in general unbounded and we shall not use it.

We denote by $p(a)=r\left(a^{*} a\right)^{1 / 2}$ the Pták function of an element $a \in A$.

${ }^{1}$ The research has been supported by a grant from La Junta de Andalucía and by the Department of Applied Mathematics, University of Seville. 
Clearly $p(a)=r\left(a^{2}\right)^{1 / 2}=r(a)$ for every selfadjoint element of $A$. As $r\left(a^{*} a\right)=$ $\max \left\{|\lambda|, \lambda \in \sigma_{\tilde{A}}\left(a^{*} a\right)\right\}=\max \left\{|\lambda|, \lambda \in \sigma_{\tilde{A}}\left(a a^{*}\right)\right\}=r\left(a a^{*}\right)$, we have $p\left(a^{*}\right)=p(a)$ for every $a \in A$. On the other hand in general $r\left(a^{*}\right)$ is not equal to $r(a)$. The set of all selfadjoint elements of $A$ will be denoted by $H(A)$. We shall write $a \geq 0$ if $a^{*}=a$ and $\left.\sigma_{\tilde{A}}(a) \subset<0, \infty\right)$.

Let us recall the basic properties of Hermitian Banach algebras. From a great number of equivalent conditions (see [9], Theorem 5,10) we choose the most important:

Theorem: Let $A$ be a Banach $*$-algebra. Then the following conditions are equivalent:

1) $A$ is a Hermitian Banach algebra (i.e. $\sigma(a) \subset \mathbf{R}$ for all selfadjoint $a \in A$ )

2) $r(x) \leq p(x)$ for all $x \in A$

3) $p(x+y) \leq p(x)+p(y)$ for all $x, y \in A$

4) $1+x^{*} x$ is invertible for all $x \in A$.

In this paper we shall use the following definition:

Definition: Let $A$ be a normed $*$-algebra. We say that $A$ is an $H$-algebra if $r(x+y) \leq$ $p(x)+p(y)$ for all $x, y \in A$.

Clearly, the condition $r(x+y) \leq p(x)+p(y)$ implies $r(x) \leq p(x)$ by setting $y=0$. On the other hand $r(x+y) \leq p(x)+p(y)$ is an immediate consequence of properties 2) and 3) in the theorem above. Thus a Banach $*$-algebra is Hermitian if and only if it is an $\mathrm{H}$-algebra. From this point of view (and also taking into account the subsequent results), $H$-algebras deserve to be called Hermitian. We were afraid, however, that this notation might be a little bit confusing.

Remark 1. Let $\tau_{A}(a)$ be the approximate point spectrum (see [5]) of an element $a \in A$, i.e. $\tau_{A}(a)=\tau_{A}^{(l)} \cup \tau_{A}^{(r)}$, where

$$
\begin{aligned}
\tau_{A}^{(l)}(a) & =\{\lambda \in \mathbf{C}, \inf \{\|(a-\lambda) x\|, x \in A,\|x\|=1\}=0\} \text { and } \\
\tau_{A}^{(r)}(a) & =\{\lambda \in \mathbf{C}, \inf \{\|x(a-\lambda)\|, x \in A,\|x\|=1\}=0\} .
\end{aligned}
$$

It is easy to see that $\tau_{A}^{(l)}(a)=\tau_{\tilde{A}}^{(l)}(a)$ and $\tau_{A}^{(r)}(a)=\tau_{\tilde{A}}^{(r)}(a)$.

It is well - known that $\delta \sigma_{\tilde{A}}(a) \subset \tau_{\tilde{A}}^{(l)}(a) \cap \tau_{\tilde{A}}^{(r)}(a)$ and $\tau_{\tilde{A}}^{(l)}(a) \cup \tau_{\tilde{A}}^{(r)}(a) \subset \sigma_{\tilde{A}}(a)$. So $\hat{\tau}_{A}^{(l)}(a)=\hat{\tau}_{A}^{(r)}(a)=\hat{\tau}_{A}(a)=\hat{\sigma}_{\tilde{A}}(a)$, where $\hat{M}$ denotes the polynomially convex hull of $M$. This means that $\sigma_{\tilde{A}}(a)$ can be replaced by $\tau_{A}(a)$ (or by $\tau_{A}^{(l)}(a), \tau_{A}^{(r)}(a)$,respectively) in all conditions that will be considered later. For example $a \geq 0$ if and only if $a^{*}=a$ and $\left.\tau_{A}(a) \subset<0, \infty\right)$. The approximate point spectrum has the aesthetic advantage that it is not necessary to go out of the algebra $A$ to the define it. On the other hand the spectrum $\sigma_{\tilde{A}}(a)$ in the Banach algebra $\widetilde{A}$ is more conveniente to use, therefore in the following we shall stick to it. 
Theorem 1: Let $A$ be a normed *-algebra satisfying $r(x) \leq p(x)$ for every $x \in A$. Then

1) $\sigma_{\tilde{A}}(a) \subset \mathbf{R}$ for every $a \in H(A)$

2) if $a, b \in H(A)$ then $r(a b) \leq r(a) r(b)$

3) $p(x y) \leq p(x) p(y)$ for all $x, y \in A$

4) $r(x)=r\left(x^{*}\right)=\lim _{n \rightarrow \infty} p\left(x^{n}\right)^{1 / n}=\inf _{n} p\left(x^{n}\right)^{1 / n}$ for all $x \in A$.

PROOF.

1) Suppose that there exists $a \in H(A)$ and $\alpha+i \beta \in \sigma_{\tilde{A}}(a)$, where $\alpha, \beta \in \mathbf{R}, \beta \neq 0$. Then, for every real $t$, we have

$$
\alpha+i \beta+i t \in \sigma_{\tilde{A}}(a+i t) \text {, i.e. } r(a+i t)^{2} \geq \alpha^{2}+(\beta+t)^{2} .
$$

On the other hand

$$
p(a+i t)^{2}=r((a-i t)(a+i t))=r\left(a^{2}+t^{2}\right) \leq r\left(a^{2}\right)+t^{2} .
$$

Thus

$$
\begin{aligned}
(\beta+t)^{2} & \leq r(a+i t)^{2} \leq p(a+i t)^{2} \leq r\left(a^{2}\right)+t^{2}, \\
2 \beta t+\beta^{2} & \leq r\left(a^{2}\right)
\end{aligned}
$$

for every real $t$. Hence $\beta=0$, a contradiction.

2) Let $a, b \in H(A)$. Then

$$
r(a b) \leq p(a b)=r(b a a b)^{1 / 2}=r\left(a^{2} b^{2}\right)^{1 / 2} .
$$

By the induction we get

$$
r(a b) \leq r\left(a^{2^{k}} b^{2^{k}}\right)^{1 / 2^{k}} \leq\left\|a^{2^{k}}\right\|^{1 / 2^{k}}\left\|b^{2^{k}}\right\|^{1 / 2^{k}} \longrightarrow r(a) r(b) \text { as } k \rightarrow \infty .
$$

3) Let $x, y \in A$. Then

$$
p(x y)=r\left(y^{*} x^{*} x y\right)^{1 / 2}=r\left(x^{*} x y y^{*}\right)^{1 / 2} \leq r\left(x^{*} x\right)^{1 / 2} r\left(y y^{*}\right)^{1 / 2}=p(x) p(y) .
$$

4) By 3) we have $p\left(x^{n+m}\right) \leq p\left(x^{n}\right) p\left(x^{m}\right)$ for every positive integers $n, m$. It is wellknown that this implies that the limit $\lim _{n \rightarrow \infty} p\left(x^{n}\right)^{1 / n}$ exists and $\lim _{n \rightarrow \infty} p\left(x^{n}\right)^{1 / n}=$ $\inf _{n} p\left(x^{n}\right)^{1 / n}$. We have $p\left(x^{n}\right)^{1 / n}=r\left(x^{* n} x^{n}\right)^{1 / 2 n} \leq\left\|x^{* n}\right\|^{1 / 2 n}\|x\|^{1 / 2 n}$, hence $\lim _{n \rightarrow \infty} p\left(x^{n}\right)^{1 / n} \leq r\left(x^{*}\right)^{1 / 2} r(x)^{1 / 2}$. Further $r(x)=r\left(x^{n}\right)^{1 / n} \leq p\left(x^{n}\right)^{1 / n}$, and so $r(x) \leq \lim _{n \rightarrow \infty} p\left(x^{n}\right)^{1 / n}$. Similarly $r\left(x^{*}\right) \leq \lim _{n \rightarrow \infty} p\left(x^{* n}\right)^{1 / n}=\lim _{n \rightarrow \infty} p\left(x^{n}\right)^{1 / n}$. Thus

$$
\max \left\{r(x), r\left(x^{*}\right)\right\} \leq \lim _{n \rightarrow \infty} p\left(x^{n}\right)^{1 / n} \leq r\left(x^{*}\right)^{1 / 2} r(x)^{1 / 2} .
$$


Hence $r\left(x^{*}\right)=r(x)=\lim _{n \rightarrow \infty} p\left(x^{n}\right)^{1 / n}$.

Theorem 2: Let $A$ be a normed $*$-algebra. Suppose that $\sigma_{\tilde{A}}(a) \subset \mathbf{R}$ for every $a \in H(A)$, and $a+b \geq 0$ whenever $a, b \in A, a \geq 0, b \geq 0$. Then

1) If $a, b \in H(A)$ then $r(a+b) \leq r(a)+r(b)$

2) $r\left(x^{*}+x\right) \leq 2 p(x)$ for every $x \in A$

3) $x^{*} x \geq 0$ for every $x \in A$.

\section{PROOF.}

1) Let $\operatorname{conv} \sigma_{\tilde{A}}(a)=\left\langle\alpha, \beta>\right.$ and $\sigma_{\tilde{A}}(b)=<\gamma, \delta>$. Then $a-\alpha \geq 0$ and $b-\gamma \geq 0$, so $(a+b)-(\alpha+\gamma) \geq 0$, i.e. $\left.\sigma_{\tilde{A}}(a+b) \subset<\alpha+\gamma, \infty\right)$. Similarly $\beta-a \geq 0$ and $\delta-\beta \geq 0$, so $(\beta+\delta)-(a+b) \geq 0$ and $\sigma_{\tilde{A}}(a+b) \subset\langle\alpha+\gamma, \beta+\delta>$. Hence $r(a+b) \leq \max \{|\alpha+\gamma|,|\beta+\delta|\} \leq \max \{|\alpha|,|\beta|\}+\max \{|\gamma|,|\delta|\}=r(a)+r(b)$.

$2)$ Let $x=a+i b$ where $a, b \in H(A)$. Then $x^{*}=a-i b$ and $x^{*} x+x x^{*}=2\left(a^{2}+b^{2}\right)$. As $r\left(a^{2}+b^{2}\right)-a^{2}-b^{2} \geq 0$ and $b^{2} \geq 0$, we conclude that $r\left(a^{2}+b^{2}\right)-a^{2} \geq 0$, i.e. $r\left(a^{2}\right) \leq r\left(a^{2}+b^{2}\right)$. Hence

$r\left(x^{*}+x\right)^{2}=r(2 a)^{2}=4 r\left(a^{2}\right) \leq 4 r\left(a^{2}+b^{2}\right)=2 r\left(x^{*} x+x x^{*}\right) \leq 2\left(r\left(x^{*} x\right)+r\left(x x^{*}\right)\right)=4 r\left(x^{*} x\right)=4 p(x)^{2}$.

3) By the assumption $\sigma_{\tilde{A}}\left(x^{*} x\right) \subset \mathbf{R}$ for every $x \in A$ since $x^{*} x \in H(A)$. Suppose on the contrary that there exists an element $x \in A$ and $\lambda \in \sigma_{\tilde{A}}\left(x^{*} x\right), \lambda<0$. Denote by $K=r\left(x^{*} x\right) \geq|\lambda|>0$. Let $f:<-K, K>\rightarrow \mathbf{R}$ be the function defined by

$$
\begin{array}{ll}
f(t)=\frac{3}{\sqrt{|\lambda|}} & (-K \leq t \leq 0), \\
f(t)=\frac{1}{\sqrt{t-\lambda / 9}} & (0 \leq t \leq K) .
\end{array}
$$

Clearly, $f$ is a continuous function on $<-K, K>$. Denote by $\delta=\min \left\{\frac{1}{\sqrt{|\lambda|}}, \inf _{0<t \leq K}\left\{\frac{1}{\sqrt{t}}-\frac{1}{\sqrt{t-\lambda / 9}}\right\}\right\}$. We have $\delta>0$ since $\lim _{t \rightarrow 0_{+}}\left(\frac{1}{\sqrt{t}}-\frac{1}{\sqrt{t-\lambda / 9}}\right)=+\infty$ (in fact it is easy but rather tedious to show that $\left.\delta=\frac{1}{\sqrt{K}}-\frac{1}{\sqrt{K-\lambda / 9}}\right)$. By the Stone-Weierstrass theorem there exists a polynomial $q(t)$ with real coefficients such that

$$
|q(t)-f(t)|<\delta \quad(-K \leq t \leq K) .
$$

Then $|q(t)| \leq f(t)+\delta \leq \frac{1}{\sqrt{t}}$ for $0<t \leq K$ and

$$
q(t) \geq f(t)-\delta \geq \frac{3}{\sqrt{|\lambda|}}-\frac{1}{\sqrt{|\lambda|}}=\frac{2}{\sqrt{|\lambda|}} \text { for } \quad-K \leq t \leq 0 .
$$

Consider the element $y=x q\left(x^{*} x\right) \in A$. Then

$$
y^{*} y=x^{*} x q\left(x^{*} x\right)^{2}=q_{1}\left(x^{*} x\right), \text { where } \quad q_{1}(t)=t q(t)^{2} .
$$


Clearly $q_{1}(t) \leq 0$ for $t \leq 0$ and $q_{1}(t)=t q(t)^{2} \leq t\left(\frac{1}{\sqrt{t}}\right)^{2}=1$ for $0<t \leq K$. Therefore $\sigma_{\tilde{A}}\left(y^{*} y\right) \quad=$ $\left\{q_{1}(\mu), \mu \in \sigma_{\tilde{A}}\left(x^{*} x\right)\right\} \subset\left(-\infty, 1>\right.$. Let $y=a+i b$ where $a, b \in H(A)$. Then $y^{*}=$ $a-i b$ and $y^{*} y+y y^{*}=2 a^{2}+2 b^{2} \geq 0$. Further $1-y^{*} y \geq 0$ and $1+y y^{*}=\left(1-y^{*} y\right)+$ $\left(y^{*} y+y y^{*}\right) \geq 0$. Thus $\left.\sigma_{\tilde{A}}\left(y y^{*}\right) \subset<-1, \infty\right)$. Since $\sigma_{\tilde{A}}\left(y^{*} y\right)-\{0\}=\sigma_{\tilde{A}}\left(y y^{*}\right)-\{0\}$, we conclude that $\sigma_{\tilde{A}}\left(y^{*} y\right) \subset<-1,1>$. On the other hand $q_{1}(\lambda) \in \sigma_{\tilde{A}}\left(y^{*} y\right)$ where $q_{1}(\lambda)=\lambda q(\lambda)^{2} \leq \lambda\left(\frac{2}{\sqrt{|\lambda|}}\right)^{2}=-4$, a contraction. Hence $x^{*} x \geq 0$ for every $x \in A$.

Theorem 3: Let $A$ be a normed $*$-algebra. Then the following conditions are equivalent:

1) $A$ is an $H$-algebra (i.e. $r(x+y) \leq p(x)+p(y)$ for all $x, y \in A)$.

2) $r(x) \leq p(x)$ for every $x \in A$ and $a+b \geq 0$ whenever $a, b \in A, a \geq 0, b \geq 0$

3) $r(x) \leq p(x)$ for every $x \in A$ and $p(x+y) \leq p(x)+p(y)$ for all $x, y \in A$.

\section{PROOF.}

$1 \Rightarrow 2$ : If we put $y=0$ we get $r(x) \leq p(x)$ for every $x \in A$. Also, $r(x+y) \leq$ $p(x)+p(y)=r(x)+r(y)$ for all $x, y \in H(A)$. Let $a, b \in A, a \geq 0, b \geq 0$. Set $c=r(a)-a, d=r(b)-b$. Clearly $c, d \in H(A), \sigma_{\tilde{A}}(c) \subset<0, r(a)>$ and $\sigma_{\tilde{A}}(d) \subset<$ $0, r(b)>$, so that $r(c) \leq r(a), r(d) \leq r(b)$ and $r(c+d) \leq r(c)+r(d) \leq r(a)+r(b)$. Hence $a+b=r(a)+r(b)-(c+d) \geq 0$.

$2 \Rightarrow 3$ : Let $x, y \in A$. By using Theorems 1 and 2 we get $p(x+y)^{2}=r((x+$ $\left.y)^{*}(x+y)\right) \leq r\left(x^{*} x\right)+r\left(y^{*} y\right)+r\left(x^{*} y+y^{*} x\right) \leq p(x)^{2}+p(y)^{2}+2 p\left(x^{*} y\right) \leq p(x)^{2}+$ $p(y)^{2}+2 p\left(x^{*} y\right) \leq p(x)^{2}+p(y)^{2}+2 p\left(x^{*}\right) p(y)=(p(x)+p(y))^{2}$.

$3 \Rightarrow 1$ : We have $r(x+y) \leq p(x+y) \leq p(x)+p(y)$.

Notation. Let $A$ be a normed $*$-algebra. We denote by $P(A)$ the set of all linear functionals $f \in A^{\prime}$ satisfying $f(1)=1$ and $|f(x)| \leq p(x)$ for every $x \in A$.

Theorem 4: Let $A$ be an $H$-algebra and let $f \in P(A)$. Then

1) $f(a) \in \mathbf{R}$ for every $a \in H(A)$

2) $f(a) \in \operatorname{conv} \sigma_{\tilde{A}}(a)$ for every $a \in H(A)$

3) $f\left(x^{*} x\right) \geq 0$ for every $x \in A$

4) $f\left(x^{*} y\right)=\overline{f\left(y^{*} x\right)}$ for all $x, y \in A$

5) $\left|f\left(x^{*} y\right)\right|^{2} \leq f\left(x^{*} x\right) f\left(y^{*} y\right)$ for all $x, y \in A$, in particular $|f(y)| \leq f\left(y^{*} y\right)^{1 / 2}$ for all $y \in A$

6) $f\left(x^{*} y^{*} y x\right) \leq f\left(x^{*} x\right) r\left(y^{*} y\right)$ for all $x, y \in A$.

\section{PROOF.}

1) Let $a \in H(A), f \in P(A)$ and let $f(a)=\alpha+i \beta$ with $\alpha, \beta \in \mathbf{R}$. For every $t \in \mathbf{R}$ we have

$$
|f(a+i t)|^{2}=|\alpha+i \beta+i t|^{2}=\alpha^{2}+(\beta+t)^{2}
$$

and

$$
p(a+i t)^{2}=r((a-i t)(a+i t))=r\left(a^{2}+t^{2}\right) \leq r\left(a^{2}\right)+t^{2} .
$$


Thus $\alpha^{2}+\beta^{2}+2 \beta t \leq r\left(a^{2}\right)$ for every $t \in \mathbf{R}$, hence $\beta=0$ and $f(a) \in \mathbf{R}$.

2) We have $|f(b)| \leq p(b)=r(b)$ for every $b \in H(A)$. Let $a \in H(A)$ and let $\operatorname{conv} \sigma_{\tilde{A}}(a)=<\alpha, \beta>$. Then $f(a)-\alpha=f(a-\alpha) \leq r(a-\alpha)=\beta-\alpha$ since conv $\sigma_{\tilde{A}}(a-\alpha)=<0, \beta-\alpha>$. So $f(a) \leq \beta$ and similarly

$$
\beta-f(a)=f(\beta-a) \leq r(\beta-a)=\beta-\alpha, \text { so } f(a) \geq \alpha .
$$

Hence $f(a) \in<\alpha, \beta>=\operatorname{conv} \sigma_{\tilde{A}}(a)$.

3) Follows immediately from 2) since $x^{*} x \geq 0$ for every $x \in A$.

4) Let $x, y \in A$ and let $\mu$ be an arbitrary complex number. Then by 3 ) we have

$$
0 \leq f\left((x+\mu y)^{*}(x+\mu y)\right)=f\left(x^{*} x\right)+\bar{\mu} f\left(y^{*} x\right)+\mu f\left(x^{*} y\right)+|\mu|^{2} f\left(y^{*} y\right)
$$

so that $\bar{\mu} f\left(y^{*} x\right)+\mu f\left(x^{*} y\right)$ is real for every $\mu \in \mathbf{C}$. For $\mu=1$ we get $\operatorname{Im} f\left(y^{*} x\right)+$ $\operatorname{Im} f\left(x^{*} y\right)=0$ and if $\mu=i$ then $\operatorname{Re} f\left(y^{*} x\right)=\operatorname{Re} f\left(x^{*} y\right)$. It follows that $f\left(x^{*} y\right)=$ $\overline{f\left(y^{*} x\right)}$.

5) For $t$ real set $\mu=t f\left(y^{*} x\right)$. The inequality above then gives

$$
0 \leq f\left(x^{*} x\right)+2 t\left|f\left(x^{*} y\right)\right|^{2}+t^{2}\left|f\left(x^{*} y\right)\right|^{2} f\left(y^{*} y\right)
$$

for every $t \in \mathbf{R}$, i.e.

$$
4\left|f\left(x^{*} y\right)\right|^{4}-4 f\left(x^{*} x\right)\left|f\left(x^{*} y\right)\right|^{2} f\left(y^{*} y\right) \leq 0 .
$$

Hence $\left|f\left(x^{*} y\right)\right|^{2} \leq f\left(x^{*} x\right) f\left(y^{*} y\right)$.

Setting $x=1$, we get, in particular,

$$
|f(y)| \leq f\left(y^{*} y\right)^{1 / 2} .
$$

6) If $f\left(x^{*} x\right)=0$ then, by 5$)$,

$$
\left|f\left(x^{*} y^{*} y x\right)\right|^{2} \leq f\left(x^{*} x\right) f\left(z^{*} z\right) \text { for } z=y^{*} y x,
$$

so that the inequality is satisfied trivially. Suppose $f\left(x^{*} x\right)>0$. Let $k$ be a nonnegative integer. Inequality 5) for the pair of vectors $x$ and $\left(y^{*} y\right)^{2^{k}} x$ gives

$$
f\left(x^{*}\left(y^{*} y\right)^{2^{k}} x\right)^{2} \leq f\left(x^{*} x\right) f\left(x^{*}\left(y^{*} y\right)^{2^{k+1}} x\right) .
$$

Thus

$$
\begin{aligned}
& f\left(x^{*} y^{*} y x\right)^{2^{n}} \leq f\left(x^{*} x\right)^{2^{n-1}} f\left(x^{*}\left(y^{*} y\right)^{2} x\right)^{2^{n-1}} \leq \\
\leq & f\left(x^{*} x\right)^{2^{n-1}} f\left(x^{*} x\right)^{2^{n-2}} \ldots f\left(x^{*} x\right) f\left(x^{*}\left(y^{*} y\right)^{2^{n}} x\right) \leq \\
\leq & f\left(x^{*} x\right)^{2^{n}-1} r\left(x^{*}\left(y^{*} y\right)^{2^{n}} x\right) \leq f\left(x^{*} x\right)^{2^{n}} \frac{r\left(x x^{*}\right)}{f\left(x^{*} x\right)} r\left(\left(y^{*} y\right)^{2^{n}}\right)
\end{aligned}
$$


(we used the submultiplicivity of the spectral radius or $H(A)$ ). Hence

$$
f\left(x^{*} y^{*} y x\right) \leq f\left(x^{*} x\right)\left(\frac{r\left(x x^{*}\right)}{f\left(x^{*} x\right)}\right)^{1 / 2^{n}} r\left(y^{*} y\right) \rightarrow f\left(x^{*} x\right) r\left(y^{*} y\right)
$$

as $n \rightarrow \infty$ (note that $\left.r\left(x x^{*}\right)=r\left(x^{*} x\right) \geq f\left(x^{*} x\right)>0\right)$.

Theorem 5: Let $A$ be an $H$-algebra and let $f \in P(A)$. Then there exist a Hilbert space $H_{f}$, a vector $\xi_{f} \in H_{f}$ and a $*$-representation $\pi_{f}: A \rightarrow B\left(H_{f}\right)$ such that

1) $f(a)=<\pi_{f}(a) \xi_{f}, \xi_{f}>$ for alll $a \in A$

2) $f\left(a^{*} a\right)^{1 / 2} \leq\left\|\pi_{f}(a)\right\| \leq p(a)$ for all $a \in A$.

PROOF. The construction of the Hilbert space $H_{f}$ is standart, therefore we give only an outline of the proof. For details see e.g. [9], Theorem 2.5. Define a semidefinite scalar product in $A$ by $\langle a, b\rangle=f\left(b^{*} a\right)$ and the corresponding norm $\|a\|_{f}^{2}=<a, a>$. Denote by $L_{f}=\left\{a \in A,\|a\|_{f}=0\right\}=\left\{a \in A, f\left(a^{*} a\right)=0\right\}$. It is easy to see by the previous theorem that $L_{f}$ is a left ideal in $A$. Consider the quotient space $A \mid L_{f}$ and denote its completion by $A_{f}$. Then $H_{f}$ is a Hilbert space.

For every $a \in A$, define $\pi_{f}(a): A\left|L_{f} \rightarrow A\right| L_{f}$ by $\pi_{f}(a)\left(x+L_{f}\right)=a x+L_{f}$. The definition is correct and

$$
\frac{\left\|\pi_{f}(a)\left(x+L_{f}\right)\right\|_{f}^{2}}{\left\|x+L_{f}\right\|_{f}^{2}}=\frac{f\left(x^{*} a^{*} a x\right)}{f\left(x^{*} x\right)} \leq r\left(a^{*} a\right)=p(a)^{2},
$$

so that $\pi_{a}(f)$ is continuous and there is a unique extension of $\pi_{f}(a)$ to an operator on $H_{f}$. Further

$<\pi_{f}(a)\left(x+L_{f}\right), y+L_{f}>=f\left(y^{*} a x\right)=<x+L_{f}, a^{*} y+L_{f}>=<x+L_{f}, \pi_{f}\left(a^{*}\right)\left(y+L_{f}\right)>$ hence $\pi_{f}\left(a^{*}\right)=\pi_{f}(a)^{*}$ and $\pi_{f}$ is a $*$-representation.If we put $\xi_{f}=1_{A}+L_{f}$ we have 1) and

$$
\left\|\pi_{f}(a)\right\|^{2} \geq\left\|\pi_{f}(a)\left(1+L_{f}\right)\right\|_{f}^{2}=f\left(a^{*} a\right) \text { for all } a \in A .
$$

Theorem 6: Let $A$ be an $H$-algebra and let $a \in H(A)$. Then

$$
\{f(a), f \in P(A)\}=\operatorname{conv} \sigma_{\tilde{A}}(a) .
$$

PROOF. By Theorem 4, $\{f(a), f \in P(A)\} \subset \operatorname{conv} \sigma_{\tilde{\tilde{A}}}(a)$. Let $a \in H(A)$. Denote by $A_{0}$ the smallest algebra containing $a$ and 1 . Let $\widetilde{A}_{0}$ be the closure of $A_{0}$ in $\tilde{A}$. Let $\lambda \in \operatorname{conv} \sigma_{\tilde{A}}(a)$. Since $\widetilde{A}_{0}$ is a commutative Banach algebra with one generator $a$, we have $\sigma_{\tilde{A}_{0}}(a)=\operatorname{conv} \sigma_{\tilde{A}}(a) \ni \lambda$ and there exists a multiplicative functional $g: \widetilde{A}_{0} \rightarrow \mathbf{C}$ with $g(a)=\lambda$. Let $x \in A_{0}$, i.e. $x=\sum_{i=0}^{n} \alpha_{i} a^{i}$ for some complex coefficients $\alpha_{i}$. Then

$$
x^{*}=\sum_{i=0}^{n} \bar{\alpha}_{i} a^{i} \text { and } g\left(x^{*}\right)=\sum_{i=0}^{n} \bar{\alpha}_{i} \lambda^{i}=\overline{\sum_{i=0}^{n} \alpha_{i} \lambda^{i}}=\overline{g(x)} .
$$


It follows that $|g(x)|^{2}=\overline{g(x)} g(x)=g\left(x^{*}\right) g(x)=g\left(x^{*} x\right) \in \sigma_{\tilde{A}_{0}}\left(x^{*} x\right)$. Hence $|g(x)| \leq r\left(x^{*} x\right)^{1 / 2}=p(x)$ for every $x \in A_{0}$. Since $p$ is a seminorm, by the HahnBanach theorem there exists an extension $f \in A^{\prime}$ with

$$
|f(y)| \leq p(y) \text { for every } y \in A \text { and } f\left|A_{0}=g\right| A_{0} \text {. }
$$

In particular, $f(1)=1$, and so $f \in P(A)$ and $f(a)=\lambda$.

Theorem 7: Let $A$ be an $H$-algebra. Then there exists a Hilbert space $H$ and a *representation

$\pi: A \rightarrow B(H)$ such that $\|\pi(x)\|=p(x)$ for every $x \in A$. Further $r(\pi(x))=r(x)$ and $\hat{\sigma}_{B(H)}(\pi(x))=\hat{\sigma}_{\tilde{A}(x)}$ for all $x \in A$.

PROOF. Let $H=\bigoplus_{f \in P(A)} H_{f}$ and $\pi=\bigoplus_{f \in P(A)} \pi_{f}$, where $H_{f}$ and $\pi_{f}$ are constructed in Theorem 5.

For every $x \in H$ we have $\|\pi(x)\|=\sup _{f \in P(A)}\left\|\pi_{f}(x)\right\|$, where $f\left(x^{*} x\right)^{1 / 2} \leq\left\|\pi_{f}(x)\right\| \leq$ $p(x)$. Since $\sup _{f \in P(A)} f\left(x^{*} x\right)^{1 / 2}=r\left(x^{*} x\right)^{1 / 2}=p(x)$ by Theorem 6 we have $\|\pi(x)\|=$ $p(x)$ for every $x \in A$. By Theorem 1 we have $r(x)=\lim _{n \rightarrow \infty} p\left(x^{n}\right)^{1 / n}=\lim _{n \rightarrow \infty}\left\|\pi\left(x^{n}\right)\right\|^{1 / n}=$ $\lim _{n \rightarrow \infty}\left\|\pi(x)^{n}\right\|^{1 / n}=r(\pi(x))$ for all $x \in A$. For every polynomial $q$ we have

$$
\max \left\{|q(\mu)|, \mu \in \sigma_{B(H)}(\pi(x))\right\}=r(q(\pi(x)))=r(q(x))=\max \left\{|q(\mu)|, \mu \in \sigma_{\tilde{A}}(x)\right\} .
$$

Hence $\lambda \in \hat{\sigma}_{B(H)}(\pi(x))$ if and only if $\lambda \in \hat{\sigma}_{\tilde{A}}(x)$.

Theorem 8: Let $A$ be a normed *-algebra. Then the following conditions are equivalent:

1) $A$ is an $H$-algebra (i.e. $r(x+y) \leq p(x)+p(y)$ for all $x, y \in A$ )

2) $r(x) \leq p(x)$ and $a+b \geq 0$ whenever $a, b \in A, a \geq 0, b \geq 0$.

3) $r(x) \leq p(x)$ and $p(x+y) \leq p(x)+p(y)$

4) There exists a Hilbert space $H$ and a $*$-representation $\pi \cdot A \rightarrow B(H)$ such that $r(\pi(x))=r(x)$ for every $x \in A$

5) $1+a+b+i c$ is invertible in $\widetilde{A}$ for every $a, b, c \in H(A), a \geq 0, b \geq 0$

6) $1+a+b+i c$ is not a left (right) topological division of zero in $A$ for all $a, b, c \in$ $H(A), a \geq 0, b \geq 0$.

PROOF. The equivalence of the first three condition was proved in Theorem 3 . The implication $1 \Rightarrow 4$ is contained in Theorem 7 .

$4 \Rightarrow 5:$ As in the previous theorem we have $\hat{\sigma}_{\tilde{A}}(x)=\hat{\sigma}_{B(H)}(\pi(x))$ for all $x \in A$. In particular,

$$
\begin{gathered}
\hat{\sigma}_{\tilde{A}}(a+b+i c)=\hat{\sigma}_{B(H)}((\pi(a+b+i c)) \subset W(\pi(a)+\pi(b)+i \pi(c)) \subset \\
\subset W(\pi(a))+W(\pi(b))+i W(\pi(c)) \subset<0, \infty)+<0, \infty)+i \mathbf{R} \subset\{z \in \mathbf{C}, \text { Re } z \geq 0\}
\end{gathered}
$$


where $W(T)$ is the numerical range of an operator $T \in B(H)$; it is well-known that $W(T)$ is a convex set containing the spectrum and $W(S)=\operatorname{conv} \sigma_{B(H)}(S)$ for every selfadjoint operator $S$. Therefore $-1 \notin \sigma_{\tilde{A}}(a+b+i c)$, i.e. $1+a+b+i c$ is invertible in $\widetilde{A}$.

$5 \Rightarrow 2$ : First we show that 5) implies $\operatorname{Re} \sigma_{\tilde{A}}(a+b+i c) \geq 0$ for all $a, b, c \in$ $H(A), a \geq 0, b \geq 0$. Indeed, if $\alpha+i \beta \in \sigma_{\tilde{A}}(a+b+i c)$ with $\alpha, \beta \in \mathbf{R}, \alpha<0$ then $-1 \in \sigma_{\tilde{A}}\left(\frac{a}{|\alpha|}+\frac{b}{|\alpha|}+i \frac{c-\beta}{|\alpha|}\right)$, a contradiction. Setting $a=b=0$ we get $\operatorname{Re} \sigma_{\tilde{A}}(-i c) \geq 0$, hence $\sigma_{\tilde{A}}(c) \subset \mathbf{R}$ for every $c \in H(A)$.

Setting $c=0$ we get that $a \geq 0$ and $b \geq 0$ implies $a+b \geq 0$. To prove $r(x) \leq p(x)$ it is sufficient to show that $\lambda \in \mathbf{C},|\lambda|>p(x)$ implies that $\lambda-x$ is invertible in $\tilde{A}$. We have

$$
\begin{aligned}
& \left(\bar{\lambda}+x^{*}\right)(\lambda-x)=|\lambda|^{2}-x^{*} x+\lambda x^{*}-\bar{\lambda} x= \\
= & \left(|\lambda|^{2}-p(x)^{2}\right)+\left(p(x)^{2}-x^{*} x\right)+i\left(i \bar{\lambda} x-i \lambda x^{*}\right),
\end{aligned}
$$

where $p(x)^{2}-x^{*} x \geq 0$ and $i \bar{\lambda} x-i \lambda x^{*} \in H(A)$. So $\operatorname{Re} \sigma_{\tilde{A}}\left(p(x)^{2}-x^{*} x+i(i \bar{\lambda} x-\right.$ $\left.\left.i \lambda x^{*}\right)\right) \geq 0$ and $\operatorname{Re} \sigma_{\tilde{A}}\left(\left(\bar{\lambda}+x^{*}\right)(\lambda-x)\right) \geq|\lambda|^{2}-p(x)^{2}>0$, so that $\left(\bar{\lambda}+x^{*}\right)(\lambda-x)$ is invertible in $\tilde{A}$, hence also $\lambda-x$ is invertible in $\widetilde{A}$.

$5 \Leftrightarrow 6$ : We have seen above that condition 5$)$ is equivalent to the condition $\operatorname{Re} \sigma_{\tilde{A}}(a+b+i c) \geq 0$ for all $a, b, c \in H(A), a \geq 0, b \geq 0$. In the same way 6$)$ is equivalent to the condition $\operatorname{Re} \tau_{A}^{l}(a+b+i c) \geq 0 \quad\left(\operatorname{Re} \tau_{A}^{(r)}(a+b+i c) \geq 0\right)$ for all $a, b, c \in H(A), a \geq 0, b \geq 0$.

The rest follows from the fact that $\hat{\sigma}_{\tilde{A}}(a+b+i c)=\hat{\tau}_{A}^{(l)}(a+b+i c)=\hat{\tau}_{A}^{(r)}(a+b+i c)$.

Remark 2: Although the theory of Hermitian Banach algebras is already classical, the observation that $r(x)=r(\pi(x))$ for all $x \in A$ (and consequently the equivalence $4 \Leftrightarrow A$ is an $H$-algebra) seems to be new even for Hermitian Banach agebras.

In particular cases it is possible to obtain stronger results than in Theorem 8. For commutative normed $*$-algebras the situation is completely analogous to the Banach algebras case:

Theorem 9 : Let $A$ be a commutative normed $*$-algebra. Then the following conditions are equivalent:

1) $A$ is an $H$-algebra

2) $\sigma_{\tilde{A}}(a) \subset \mathbf{R}$ for all $a \in H(A)$

3) $r(x) \leq p(x)$ for all $x \in A$

4) $p(x+y) \leq p(x)+p(y)$ for all $x \in A$

5) $1+x^{*} x$ is invertible in $\tilde{A}$ for all $x \in A$

6) $1+x^{*} x$ is not a left (right) topological divisor of 0 in $A$ for all $x \in A$.

PROOF. If $A$ is an $H$-algebra then all the remaining conditions are satisfied by the previous theorem. 
$2 \Rightarrow 1:$ Let $a, b, c \in H(A), a \geq 0, b \geq 0$. Then

$\left.\left.\sigma_{\tilde{A}}(a+b+i c) \subset \sigma_{\tilde{A}}(a)+\sigma_{\tilde{A}}(b)+i \sigma_{\tilde{A}}(c) \subset<0, \infty\right)+<0, \infty\right)+i \mathbf{R} \subset\{z \in \mathbf{C}, \operatorname{Re} z \geq 0\}$ so that $1+a+b+i c$ is invertible in $\tilde{A}$ and $A$ is an $H$-algebra.

The implication $3 \Rightarrow 2$ is true in general (see Theorem 1).

$4 \Rightarrow 3$ : For $x \in A$ set $a=\frac{1}{2}\left(x+x^{*}\right)$ and $b=\frac{1}{2 i}\left(x-x^{*}\right)$. Then $x=a+i b, a, b \in$ $H(A)$ and

$$
\begin{aligned}
r(x) & \leq r(a)+r(i b)=p(a)+p(b)=\frac{1}{2} p\left(x+x^{*}\right)+\frac{1}{2} p\left(x-x^{*}\right) \leq \\
& \leq \frac{1}{2}\left(p(x)+p\left(x^{*}\right)+\frac{1}{2}\left(p(x)+p\left(x^{*}\right)\right)=2 p(x) .\right.
\end{aligned}
$$

Hence

$$
\left.r(x)^{n}=r\left(x^{n}\right) \leq 2 p\left(x^{n}\right)=2 r\left(x^{* n} x^{n}\right)^{1 / 2}=2 r\left(x^{*} x\right)^{n}\right)^{1 / 2}=2 r\left(x^{*} x\right)^{n / 2}=2 p(x)^{n} .
$$

If $n \rightarrow \infty$ then we get $r(x) \leq p(x)$.

$5 \Rightarrow 2$ : Suppose on the contrary that there exists $a \in H(A)$ and $\alpha+i \beta \in \sigma_{\tilde{A}}(a)$ with $\alpha, \beta \in \mathbf{R}, \beta \neq 0$. Then setting $x=\beta^{-1}(a-\alpha)$ we get $i \in \sigma_{\tilde{A}}(x)$ and $-1 \in \sigma_{\tilde{A}}\left(x^{2}\right)=\sigma_{\tilde{A}}\left(x^{*} x\right)$, a contradiction.

The implication $6 \Rightarrow 2$ can be proved in the same way as $\sigma_{\tilde{A}}(a) \subset \mathbf{R}$ if and only if $\tau_{A}^{(l)}(a)$

R $\left(\tau_{A}^{(r)}(a) \subset \mathbf{R}\right)$.

Other case, when it is possible to obtain stronger results is the case of algebras with open set of invertible elements, i.e. $Q$-algebra in the sense of [7]. Suppose that $A$ is a normed $Q$-algebra, i.e. there exists $\varepsilon>0$ such that $\{1-x, x \in A,\|x\|<$ $\varepsilon\} \subset \operatorname{Inv}(A)$ where $\operatorname{Inv}(A)$ denotes the set of all invertible elements in $A$. Then, for each $y \in A$ and $\lambda \in \mathbf{C}, \lambda>\frac{\|y\|}{\varepsilon}$, we have $\lambda-y=\lambda\left(1-\frac{y}{\lambda}\right) \in \operatorname{Inv}(A)$ so that

$$
\max \{|\lambda|, \lambda-y \notin \operatorname{Inv}(A)\} \leq \frac{\|y\|}{\varepsilon}
$$

Thus

$\max \{|\lambda|, \lambda-y \notin \operatorname{Inv}(A)\}=\max \left\{|\mu|, \mu-y^{n} \notin \operatorname{Inv}(A)\right\}^{1 / n} \leq\left(\frac{\left\|y^{n}\right\|}{\varepsilon}\right)^{1 / n} \rightarrow r(y)$.

Hence

$$
\max \{|\lambda|, \lambda-y \notin \operatorname{Inv}(A)\}=\max \{|\lambda|, \lambda-y \notin \operatorname{Inv}(\widetilde{A})\}=r(y)
$$

As in Theorem 7 this means that $\hat{\sigma}_{A}(y)=\hat{\sigma}_{\tilde{A}}(y)$ for all $y \in A$. 
Corollary 10: Let $A$ be a normed $*$ - $Q$-algebra. Then $A$ is an $H$-algebra if and only if $r(x) \leq p(x)$ for every $x \in A$.

PROOF. It is sufficient to show that $a+b \geq 0$ whenever $a, b \in A, a \geq 0$ and $b \geq 0$.

By Theorem $1, \sigma_{\tilde{A}}(c) \subset \mathbf{R}$ for every selfadjoint element $c \in A$. Hence it is sufficient to show that, for $a, b \in A, a \geq 0$ and $b \geq 0,-1 \notin \sigma_{\tilde{A}}(a+b)$, i.e. that $1+a+b$ is invertible in $\widetilde{A}$. Since $\left.\sigma_{A}(a) \subset \operatorname{conv} \sigma_{\tilde{A}}(a) \subset<0, \infty\right)$, we have $1+a \in \operatorname{Inv}(A)$ and similarly $1+b \in \operatorname{Inv}(A)$. We can write

$$
1+a+b=(1+a)(1+b)-a b=(1+a)(1-u v)(1+b)
$$

where $u=(1+a)^{-1} a, v=b(1+b)^{-1}$. Clearly $u, v \in H(A), r(u)<1$ and $r(v)<1$. By Theorem 1 we have $r(u v)<1$ so that $1-u v \in \operatorname{Inv}(\tilde{A})$ and also $1+a+b \in$ $\operatorname{Inv}(\tilde{A})$.

Remark 3: Suppose that $A$ is a normed $*$-algebra such that the numerical range $W(a)$ is real for every $a \in H(a)$. Then $A$ is an $H$-algebra since condition 5) of Theorem 8 is satisfied. To see this, let $a, b, c \in H(A), a \geq 0$ and $b \geq 0$. Then $\sigma_{\tilde{A}}(a+b+i c) \subset W(a+b+i c) \subset W(a)+W(b)+i W(c)=\operatorname{conv} \sigma_{\tilde{A}}(a)+\operatorname{conv} \sigma_{\tilde{A}}(b)+$ $\left.\left.i \operatorname{conv} \sigma_{\tilde{A}}(c) \subset<0, \infty\right)+<0, \infty\right)+i \mathbf{R} \subset\{z \in \mathbf{C}, \operatorname{Re} z \geq 0\}$.

By using Theorem 8 it is possible to obtain the Vidov-Palmer theorem. However, in this case the application of standart technics (see e.g. [1] p. 207) gives that the involution is continuous and therefore it can be extended to $\tilde{A}$. Thus in this case the situation reduces to the Banach algebras case. 


\section{References}

[1] F. F. Bonsall and J. Duncan: Complete normed algebras, Springer, New York 1973.

[2] J. W. M. Ford: A square root lemma for Banach star-algebras, J. London Math. Soc. 42 (1967), 521-522.

[3] I. M. Gelfand and M. A. Naimark: On the embedding of normed rings into the ring of operators on Hilbert space, Mat. Sbornik 12 (1943), 197-213.

[4] J. G. Glimm and R. V. Kadison: Unitary operators in $C^{*}$-algebras, Pacific J. Math. 10 (1960), 547-556.

[5] R. Harte: Spectral mapping theorems, Proc. Roy. Irish Acad. ser. A 72 (1972), 89-107.

[6] I. Kaplansky: Symmmetry of Banach algebras, Proc. Amer. Math. Soc. 3 (1952), 396-399.

[7] E. A. Michael: Locally multiplicately convex topological algebras, Mem. Amer. Math. Soc. No. 11 (1952).

[8] T. W. Palmer: Characterization of $C^{*}$-algebras, Bull. Amer. Math. Soc. 74 (1968), 538-540.

[9] V. Pták: Banach algebras with involution, Manuscripta Math. 6 (1972), 245290.

[10] B. Russo and H. A. Dye: A note on unitary opertors in $C^{*}$-algebras, Duke Math. J. 33 (1966), 413-416.

[11] S. Shirali and J. W. M. Ford: Symmetry in complex involutary Banach algebras, Duke Math. J. 37 (1970), 275-280.

[12] I. Vidov: Eine metrische Kennzeichnung der sellbstadjungtierten Operatoren, Math. Zeischrift 66 (1956), 121-128.

Institute of Mathematics

Czechoslovak Academy of Sciences

Žitná 25, 11567 Praha 1

Czechoslovakia 